\title{
Homoclinic chaos in the discrete self-trapping trimer
}

Hennig, D.; Gabriel, H.; Jørgensen, Michael Finn; Christiansen, Peter Leth; Clausen, Carl A. Balslev

Published in:

Physical Review E. Statistical, Nonlinear, and Soft Matter Physics

Link to article, DOI:

10.1103/PhysRevE.51.2870

Publication date:

1995

Document Version

Publisher's PDF, also known as Version of record

Link back to DTU Orbit

Citation (APA):

Hennig, D., Gabriel, H., Jørgensen, M. F., Christiansen, P. L., \& Clausen, C. A. B. (1995). Homoclinic chaos in the discrete self-trapping trimer. Physical Review E. Statistical, Nonlinear, and Soft Matter Physics, 51(4), 28702876. https://doi.org/10.1103/PhysRevE.51.2870

\section{General rights}

Copyright and moral rights for the publications made accessible in the public portal are retained by the authors and/or other copyright owners and it is a condition of accessing publications that users recognise and abide by the legal requirements associated with these rights.

- Users may download and print one copy of any publication from the public portal for the purpose of private study or research.

- You may not further distribute the material or use it for any profit-making activity or commercial gain

- You may freely distribute the URL identifying the publication in the public portal 


\title{
Homoclinic chaos in the discrete self-trapping trimer
}

\author{
D. Hennig and $\mathbf{H}$. Gabriel \\ Freie Universität Berlin, Fachbereich Physik, Institut für Theoretische Physik, Arnimallee 14, 14195 Berlin, Germany \\ M. F. Jørgensen, P. L. Christiansen, and C. B. Clausen \\ Institute of Mathematical Modelling, Technical University of Denmark, 2800 Lyngby, Denmark
}

(Received 6 September 1994)

\begin{abstract}
We study the discrete self-trapping (DST) equation with three degrees of freedom. By taking the DST dimer as the underlying unperturbed system we treat the coupling to the additional oscillator as a small perturbation. Using the generalized Melnikov method we prove the existence of homoclinic chaos in the DST-trimer dynamics.
\end{abstract}

PACS number(s): 05.45.+b

\section{INTRODUCTION}

The discrete self-trapping (DST) system is a set of coupled nonlinear (complex) oscillators, which was introduced by Eilbeck, Scott and Lomdahl [1] as a model to describe the nonlinear dynamics of small polyatomic chains such as water, ammonia, methane, acetylene, and benzene, as well as of larger molecules, such as acetanilide. The DST system arises in other fields too, e.g., quasiparticle motion on a dimer [2], stabilization of high-frequency vibrations in the field of acoustic phonons in the Davydov model [3], and in nonlinear optics to describe arrays of coupled nonlinear waveguides [4,5].

The dynamical properties and, in particular, the appearance of chaotic behavior of the DST system with a few degrees of freedom were investigated in Refs. [1,6-13]. Most of these studies were performed for the DST system with three degrees of freedom, i.e., a trimer. The DST trimer is the first nontrivial case going beyond the dimer case. The latter is integrable and the solutions can be expressed in terms of Jacobian elliptic functions $[2,14]$.

In this paper we study the integrability of the DST trimer. In a recent paper a special integrable configuration for the disordered DST trimer has been identified with the help of the Painleve analysis [15]. The solution and the localization behavior of this system has been investigated in [16]. The analysis of integrable trimer models has been extended to generalized DST systems [17]. The aim of this paper is to prove analytically the existence of chaotic solutions for the (general) DST trimer. Thereby we start from the integrable DST dimer and treat the weak coupling to the remaining third oscillator of the trimer configuration as a small perturbation. Using the Melnikov method [18-20] we prove the existence of homoclinic chaos in the perturbed dimer dynamics. This problem has also been considered by Morrison in $[21,22]$.

The paper is organized as follows. Section II presents the model for the DST trimer. In Sec. III we collect information of the DST dimer dynamics necessary to apply the Melnikov method. Section IV is devoted to the calculation of the Melnikov function and discussion of the on- set of homoclinic chaos in the coupled oscillator system. Finally, we give a short summary in Sec. V.

\section{THE DST TRIMER}

The DST trimer system is described by the Hamiltonian

$$
\begin{aligned}
H= & -\frac{1}{2} \gamma \sum_{j=1}^{3}\left|c_{j}\right|^{4}-V\left[c_{2}^{*} c_{1}+c_{1}^{*} c_{2}\right] \\
& -\epsilon\left[W_{13}\left(c_{3}^{*} c_{1}+c_{1}^{*} c_{3}\right)+W_{23}\left(c_{3}^{*} c_{2}+c_{2}^{*} c_{3}\right)\right],
\end{aligned}
$$

where $c_{j}$ are the complex field amplitudes of the $j$ th oscillator, $\gamma$ is the nonlinearity parameter, and $V, \epsilon W_{13}$, and $\epsilon W_{23}$ are the dispersion parameters determining the linear coupling between the oscillators. In this paper we consider the symmetric case $W \equiv W_{13}=W_{23}$ (closed triangular configuration). However, the asymmetric cases $W_{13} \neq W_{23}$ (asymmetric triangular configuration) and either $W_{13}=0$ or $W_{23}=0$ (open chain), could be treated as well by applying the same procedure. Letting $\epsilon=0$, i.e., $W=0$, we recover the Hamiltonian for the DST dimer. The equations of motion in the field amplitudes are derived from the Hamiltonian as follows:

$$
i \dot{c}_{j}=\frac{\partial H}{\partial c_{j}^{*}} .
$$

Besides the Hamiltonian (energy) the DST system possesses with the norm

$$
P=\sum_{j=1}^{3}\left|c_{j}\right|^{2},
$$

a further conserved quantity.

In our present investigation we use the DST dimer as a starting point for applying perturbation methods and treat thereby the coupling to a third oscillator as a small perturbation of the integrable dimer dynamics. For this reason we introduced in the Hamiltonian (1) the small positive dimensionless quantity $\epsilon<1$ to perform the transition from the trimer to the integrable dimer in the limit of vanishing $\epsilon$ as well as to emphasize the perturba- 
tive character of the coupling of an additional oscillator to the two oscillators constituting the dimer.

For an application of perturbation methods it is convenient to pass canonically to action-angle variables for the oscillator in the field amplitude $c_{3}$. Therefore we introduce

$$
c_{3}=\sqrt{J_{3}} e^{-i \theta_{3}},
$$

where $J_{3}$ is the action determining the amount of power contained in the third oscillator and $\theta_{3}$ is the corresponding canonically conjugated angle variable.

The Hamiltonian (1) expressed in these variables becomes

$$
\begin{aligned}
H^{\epsilon}= & H_{d}^{0}\left(c_{1}, c_{2}, c_{1}^{*}, c_{2}^{*}\right)+H_{3}^{0}\left(J_{3}\right) \\
& +H^{1}\left(c_{1}, c_{2}, c_{1}^{*}, c_{2}^{*}, J_{3}, \theta_{3}\right),
\end{aligned}
$$

consisting of the part for the DST dimer

$$
H_{d}^{0}=-\frac{1}{2} \gamma\left[\left|c_{1}\right|^{4}+\left|c_{2}\right|^{4}\right]-V\left[c_{1}^{*} c_{2}+c_{1} c_{2}^{*}\right],
$$

the isolated third oscillator

$$
H_{3}^{0}=-\frac{1}{2} \gamma J_{3}^{2},
$$

and the interaction part

$$
H^{1}=-\epsilon W \sqrt{J_{3}}\left\{\left(c_{1}^{*}+c_{2}^{*}\right) e^{-i \theta_{3}}+\left(c_{1}+c_{2}\right) e^{i \theta_{3}}\right\} \text {. }
$$

The corresponding set of equations of motion for the pairs of canonically conjugated variables $\left(c_{j}^{*}, c_{j}\right)$ with $j=1,2$ and $\left(J_{3}, \theta_{3}\right)$ become

$$
i \dot{c}_{j}^{*}=-\frac{\partial H}{\partial c_{j}}, \quad i \dot{c}_{j}=\frac{\partial H}{\partial c_{j}^{*}}
$$

and

$$
\dot{J}_{3}=-\frac{\partial H}{\partial \theta_{3}}, \quad \dot{\theta}_{3}=\frac{\partial H}{\partial J_{3}} .
$$

The norm is given by

$$
P=\left|c_{1}\right|^{2}+\left|c_{2}\right|^{2}+J_{3},
$$

and is the Casimir function for the Poisson brackets given above in Eqs. (9) and (10). In the integrable case, $\epsilon=0$, the system decouples into the system for the DST dimer and an isolated third oscillator with conserved action $J_{3}$. The dynamics of the DST dimer reveals a homoclinic structure for sufficiently large nonlinearity parameter $\gamma$ [14]. It is the nonintegrable destruction of this homoclinic structure as a result of the coupled trimer dynamics that we focus our interest on.

\section{THE DST DIMER AND HOMOCLINIC MANIFOLDS}

In this section we consider briefly the DST dimer in order to collect the information necessary to apply the Melnikov method to the perturbed DST dimer. For a study of the dimer dynamics it is convenient to pass from the complex-valued field amplitudes $c_{j},(j=1,2)$, to real valued variables by $\mathrm{su}(2)$ notations. Using the Pauli spin matrices $\sigma_{i}$ and Einsteins sum convention we define the following three real-valued variables $x_{i}=c_{j}^{*}\left(\sigma_{i}\right)_{j k} c_{k}$, yielding

$$
\begin{aligned}
& x_{1}=c_{2}^{*} c_{1}+c_{1}^{*} c_{2}, \\
& x_{2}=i\left[c_{2}^{*} c_{1}-c_{1}^{*} c_{2}\right], \\
& x_{3}=\left|c_{1}\right|^{2}-\left|c_{2}\right|^{2} .
\end{aligned}
$$

$N=\left|c_{1}\right|^{2}+\left|c_{2}\right|^{2}=\sqrt{x_{1}^{2}+x_{2}^{2}+x_{3}^{2}}$ is a conserved quantity in the unperturbed dimer system. In these new variables the dynamics are described by $\mathrm{SO}(3)$ rotations of a vector lying on the surface of a two-dimensional sphere $S^{2}(N)$ with radius $N=P-J_{3}$. The time evolution of the vector is governed by a (noncanonical) Lie-Poisson bracket defined on the dual space of the Lie-algebra so(3) with dual coordinates $\vec{x}=\left(x_{1}, x_{2}, x_{3}\right)$

$$
\dot{x}_{i}=2 \sum_{j=1}^{3} \Pi_{i j} \frac{\partial H_{d}^{0}}{\partial x_{j}},
$$

with the following Hamiltonian function $H_{0}^{d}: \operatorname{so}(3)^{*} \rightarrow \mathbb{R}$

$$
H_{d}^{0}=-\frac{1}{4} \gamma\left[N^{2}+x_{3}^{2}\right]-V x_{1} .
$$

The fully antisymmetric Lie-Poisson tensor $\Pi(\vec{x})$ has the following nonvanishing elements $\Pi_{12}=x_{3}, \Pi_{13}=-x_{2}$, $\Pi_{23}=x_{1}$ [23]. The system (12) and (13) written out in coordinates is

$$
\begin{aligned}
& \dot{x}_{1}=\gamma x_{2} x_{3}, \\
& \dot{x}_{2}=-\gamma x_{1} x_{3}+2 V x_{3}, \\
& \dot{x}_{3}=-2 V x_{2} .
\end{aligned}
$$

Due to the existence of two integrals of motion, namely, the energy $H_{d}^{0}=h$ and the radius of the sphere (norm) $N$, the system (14)-(16) is effectively a one-dimensional Hamiltonian system and hence completely integrable.

An analysis of the fixed points of system (14)-(16) reveals in dependence of the ratio $p=N \gamma /(2 V)$ the following structure [14].

(1) $p<1$ : Two elliptic fixed points at $x_{1}= \pm N$, $x_{2}=x_{3}=0$.

(2) $p>1$ : One elliptic fixed point at $x_{1}=-N$, $x_{2}=x_{3}=0$, one hyperbolic fixed point at $x_{1}=N$, $x_{2}=x_{3}=0$ and two elliptic fixed points at $x_{1}=2 \mathrm{~V} / \gamma$, $x_{2}=0, x_{3}= \pm \sqrt{N^{2}-x_{1}^{2}}$.

For $p>1$ a transition in the number of fixed points occurs and the stable elliptic fixed point at $x_{1}=N$, $x_{2}=x_{3}=0$ loses its stability during a pitchfork bifurcation into an unstable hyperbolic fixed point thus creating a homoclinic structure on the sphere $S^{2}(N)$ [14]. The hyperbolic point is connected to itself by a pair of homoclinic orbits, formed by the coinciding stable and unstable manifolds of the hyperbolic fixed point. The coordinates of the homoclinic orbit are given by

$x_{1}(t)=N-\frac{4 V}{\gamma} \alpha^{2} \operatorname{sech}^{2}\left[2 \alpha V\left(t-t_{0}\right)\right]$, 


$$
\begin{gathered}
x_{2}^{ \pm}(t)= \pm \frac{4 V}{\gamma} \alpha^{2} \operatorname{sech}\left[2 \alpha V\left(t-t_{0}\right)\right] \tanh \left[2 \alpha V\left(t-t_{0}\right)\right] \\
x_{3}^{ \pm}(t)= \pm \frac{4 V}{\gamma} \alpha \operatorname{sech}\left[2 \alpha V\left(t-t_{0}\right)\right]
\end{gathered}
$$

Here $t_{0}$ is the time along the unperturbed flow parametrizing the homoclinic orbit and the positive parameter $\alpha$ is related to the conserved quantity $N$ via the relation

$$
N=\sqrt{x_{1}^{2}+x_{2}^{2}+x_{3}^{2}}=\frac{2 V}{\gamma}\left(1+\alpha^{2}\right)
$$

The system (14)-(16) can be further reduced to the sphere $S^{2}$ by introducing spherical variables

$$
\begin{aligned}
& x_{1}=N \cos \phi \sin \theta, \\
& x_{2}=N \sin \phi \sin \theta, \\
& x_{3}=N \cos \theta,
\end{aligned}
$$

which are related to the complex field amplitudes via

$$
\begin{aligned}
c_{1} & =\sqrt{N} \cos \left[\frac{\theta}{2}\right] e^{-i[(\phi / 2)+\beta]} \\
& =\left[\frac{N+x_{3}}{2}\right]^{1 / 2} e^{-i[(\phi / 2)+\beta]}, \\
c_{2} & =\sqrt{N} \sin \left[\frac{\theta}{2}\right] e^{i[(\phi / 2)-\beta]}, \\
& =\left[\frac{N-x_{3}}{2}\right]^{1 / 2} e^{i[(\phi / 2)-\beta]},
\end{aligned}
$$

where $\beta$ is the angle variable conjugate to the action $N$. The reduced Hamiltonian represented in the symplectic pairs $\left(p \equiv x_{3}=N \cos \theta, \phi\right) \in S^{2} \subset \mathbb{R}^{3}$ and $(N, \beta) \in \mathbb{R}^{1} \times S^{1}(\mathrm{a}$ cylinder) reads as

$$
H_{d}^{0}=-\frac{1}{4} \gamma\left[N^{2}+p^{2}\right]-V \sqrt{N^{2}-p^{2}} \cos \phi .
$$

The Poisson bracket between two functions $F$ and $G$ is given by

$$
\{F, G\}=2\left\{\frac{\partial F}{\partial \phi} \frac{\partial G}{\partial p}-\frac{\partial F}{\partial p} \frac{\partial G}{\partial \phi}\right\}+\left\{\frac{\partial F}{\partial \beta} \frac{\partial G}{\partial N}-\frac{\partial F}{\partial N} \frac{\partial G}{\partial \beta}\right\},
$$

and the corresponding equations of motion are reproduced by substituting $(p, \phi, N, \beta)$ into the Hamiltonian form $\dot{F}=\{F, H\}$. The spherical part $c_{j} \rightarrow c_{j}(p, \phi, \cdot, \cdot)$ of the transformation given in Eqs. (24) and (25) provides a symplectic transformation with multiplier $\mu=2$ (see, e.g., Chap. IV in [24]). Therefore a factor of 2 occurs in front of the first term on the right hand side of Eq. (27). Since $\beta$ is a cyclic coordinate, the radius $N$ of the sphere $S^{2}$, being the corresponding canonically conjugated coordinate, is conserved.

For later use in Sec. IV, we note the interaction part of the Hamiltonian expressed in spherical coordinates

$$
\begin{aligned}
H^{1}=-\epsilon W \sqrt{2 J_{3}}[ & \sqrt{N+p} \cos \left(\theta_{3}-\beta-\frac{\phi}{2}\right) \\
& \left.+\sqrt{N-p} \cos \left[\theta_{3}-\beta+\frac{\phi}{2}\right]\right] .
\end{aligned}
$$

The coupled dimer-oscillation system admits a further symmetry connected with the conserved quantity $P=J_{3}+N$. The vector field associated with this integral is

$$
X_{P}=J_{3} N\left(\partial_{\theta_{3}}+\partial_{\beta}\right),
$$

which represents a simultaneous translation of the angles $\theta_{3}$ and $\beta$. This transformation leaves the Hamiltonian $H=H^{0}+H^{1}$ invariant, since it depends on the two angles only in their difference $\left[\theta_{3}-\beta\right]$. Using the $S^{1}$ symmetry (29) and introducing two pairs of canonically conjugated coordinates $\left(J_{1} \equiv J, \psi_{1} \equiv \psi\right)$ and $\left(I \equiv P, \psi_{2}\right)$, defined by the following symplectic transformations:

$$
\begin{aligned}
& J_{1}=J_{3}-N, \quad \psi_{1}=\theta_{3}-\beta, \\
& I=J_{3}+N, \quad \psi_{2}=\theta_{3}+\beta,
\end{aligned}
$$

the Hamiltonian reduces to

$$
\begin{aligned}
H^{0}= & -\frac{1}{4} \gamma\left[\frac{1}{4}(I-J)^{2}+p^{2}\right] \\
& -V\left[\frac{1}{4}(I-J)^{2}-p^{2}\right]^{1 / 2} \cos \phi-\frac{1}{8} \gamma(J+I)^{2},
\end{aligned}
$$

and

$$
\begin{aligned}
H^{1}=- & \epsilon W \sqrt{I+J} \\
\times & {\left[\left[\frac{1}{2}(I-J)+p\right]^{1 / 2} \cos \left(\psi-\frac{\phi}{2}\right)\right.} \\
& \left.+\left[\frac{1}{2}(I-J)-p\right]^{1 / 2} \cos \left(\psi+\frac{\phi}{2}\right)\right] .
\end{aligned}
$$

The Hamiltonian does not depend on the angle-variable $\psi_{2}$ and it is therefore an ignorable coordinate. Thus the system has been reduced to a two degrees of freedom Hamiltonian subsystem plus an additional quadrature for the variable $\psi_{2}$, which can be carried out once the solutions for the subsystem in the variables $(p, \phi, J, \psi)$ are known (if possible at all).

In the following we ignore the cyclic variable $\psi_{2}$ and restrict our analysis to the reduced subsystem. (An alternative approach consists in the restriction of the dynamics to a four-dimensional cross-section by fixing the phase of the angular variable $\psi_{2}^{0}$ and studying the associated Poincaré map.)

Before proceeding with an analysis of the dynamics we briefly discuss the geometrical phase space structure of the unperturbed subsystem which is four dimensional and given by $(p, \phi, J, \psi) \in\left(\mathbb{R}^{1} \times S^{1} \times \mathbb{R}^{1} \times S^{1}\right)$.

For $\gamma>2 V$ the unperturbed system has an invariant two-dimensional normally hyperbolic manifold 
$M=\left\{(p, \phi, J, \psi) \in \mathbb{R}^{1} \times S^{1} \times \mathbb{R}^{1} \times S^{1} \mid p=0, \phi=0, J \in \mathbb{R}^{+}, \psi \in[0,2 \pi)\right\}$.

This homoclinic manifold $\mathcal{M}$ has three-dimensional stable and unstable manifolds, denoted by $W_{0}^{s}(\mathcal{M})$ and $W_{0}^{u}(\mathcal{M})$, respectively. The unperturbed homoclinic orbits $\Gamma$ in the homoclinic manifold are parametrized by

$$
\begin{aligned}
& \Gamma^{ \pm}\left(t, \bar{J}, \psi^{0}, t_{0}, \alpha\right) \\
& \quad=\left(p^{ \pm, h}\left(t-t_{0}\right), \phi^{h}\left(t-t_{0}\right), J=\bar{J}, \psi^{h}(t)+\psi^{0}\right),
\end{aligned}
$$

with

$$
\begin{aligned}
& p^{ \pm, h}(t)= \pm A \operatorname{sech}(2 \alpha V t), \\
& \phi^{h}(t)=\arccos \left\{\frac{\left[1-A \alpha \operatorname{sech}^{2}(2 \alpha V t)\right]}{\sqrt{\left[1-A^{2} \operatorname{sech}^{2}(2 \alpha V t)\right.}}\right\}, \\
& \psi^{h}(t)=\int^{t} d s \omega\left(p^{ \pm, h}(s, \bar{J}), \phi^{h}(s, \bar{J}), \bar{J}\right) .
\end{aligned}
$$

where $N=\frac{1}{2}(I-J)$ and

$$
A=\frac{4 V}{\gamma N} \alpha
$$

The frequency of the decoupled third oscillator appearing in (38) is determined by $\omega=\partial H^{0} / \partial J$. Making use of Eqs. (32), (36), and (37) we obtain

$$
\begin{aligned}
\psi^{h}(t)= & -\frac{1}{8} \gamma[I+3 J] t \\
& +\frac{1}{4} V(I-J) \int_{0}^{t} d s \frac{\cos \left[\phi^{h}(s)\right]}{\left\{N^{2}-\left[p^{ \pm, h}(s)\right]^{2}\right\}^{1 / 2}},
\end{aligned}
$$

which by performing the integration for fixed action $\bar{J}$ results in

$$
\begin{aligned}
\psi^{h}(t) & =-\frac{1}{8} \gamma[I+3 \bar{J}] t \\
& +\frac{1}{4} V(I-\bar{J})\left\{\frac{1}{N} t-\left[\frac{1}{N}-\frac{\gamma}{4 V}\right] \frac{1}{\sqrt{1-A^{2}}} \frac{2}{\gamma N} \arctan \left[\left(\frac{A^{2}}{1-A^{2}}\right]^{1 / 2} \tanh (2 \alpha V t)\right]\right\} .
\end{aligned}
$$

In the unperturbed system $M$ has the structure of a oneparameter family of one-tori $T_{0}(\bar{J})$, labeled by the value of the fixed action $\bar{J}$. This one-torus has two-dimensional stable and unstable manifolds, $W_{0}^{s}\left[T_{0}(\bar{J})\right]$ and $W_{0}^{u}\left[T_{0}(\bar{J})\right]$ that are coincident along a two-dimensional homoclinic orbit.

In the next section we focus on the phenomena of homoclinic chaos in the perturbed DST dimer dynamics induced by transversal intersections of the stable and unstable manifold of the homoclinic orbit.

\section{THE MELNIKOV METHOD AND HOMOCLINIC CHAOS}

For the perturbed system, i.e., $\epsilon \neq 0$, the stable and unstable manifold of the homoclinic structure for the perturbed system may intersect transversely leading to the onset of homoclinic chaos. The analysis is based on the properties of the generalized multidimensional Melnikov function introduced by Wiggins [20]. Without going into details, we collect the main pieces of information delivered by the Melnikov method. (i) It serves to prove that the integrability of a dynamical system containing a homoclinic orbit will be broken under small perturbations by introducing homoclinic chaos into the dynamics. (ii) We learn about the separatrix splitting, i.e., the destruction of the boundary in phase space discerning between self-trapped and untrapped motion under the influence of perturbations. (iii) The Melnikov function provides a signed measure of the distance between the stable and unstable manifolds. (iv) It allows an approxi- mate location of transversal intersections of the stable and unstable manifolds along the unperturbed homoclinic orbit. By measuring analytically the area of the escape (capture) lobes of the turnstile via the Melnikov function, transport rates can be quantified [25]. (v) With the help of the Melnikov function, Holmes and Marsden [26] established a connection between the perturbation strength $\epsilon$ and the number of iterations for the Poincare map necessary to get horseshoes in the perturbed dynamics.

First of all, let us discuss briefly the geometrical structure of the perturbed phase space. Using a theorem by Fenichel [28], we see that for sufficiently small $\epsilon \neq 0$ the homoclinic manifold $\mathcal{M}$ persists as a locally invariant manifold $\mathcal{M}_{\epsilon}$ in the perturbed system and has local stable and unstable manifolds $W_{\epsilon}^{s, u}$ close to $W_{0}^{s, u}$.

Moreover, under the condition of nondegeneracy for the frequencies for the decoupled oscillators, the Kolmogorov-Arnold-Moser (KAM) theorem [29] ensures that for sufficiently small $\epsilon$ the perturbed dynamics possesses a one-parameter family of surviving invariant one-dimensional tori $T_{\epsilon}(\bar{J})$ on $\mathcal{M}_{\epsilon}$, which are again parametrized by the value of the action $\bar{J}$. The existence of the two-dimensional stable and unstable manifolds $W^{s}\left(T_{\epsilon}\right)$ and $W^{u}\left(T_{\epsilon}\right)$ of these surviving tori is guaranteed by Graff's theorem [30].

To determine whether the stable and unstable manifolds $W^{s}\left(T_{\epsilon}\right)$ and $W^{s}\left(T_{\epsilon}\right)$ of the periodic orbit $T_{\epsilon}$ intersect transversely on $M_{\epsilon}$, we have to compute the distance $d_{\epsilon}$ between the local stable and unstable manifold at each point $u^{ \pm}=\left[p^{ \pm, h}\left(-t_{0}, \alpha, \bar{J}\right), \phi^{h}\left(-t_{0}, \alpha, \bar{J}\right), \bar{J}, \psi^{0}\right]$ on the 
unperturbed homoclinic orbit $\Gamma^{ \pm}$for fixed $\left(t_{0}, \alpha, \bar{J}, \psi^{0}\right)$. We therefore introduce a moving system of homoclinic coordinates along the unperturbed homoclinic orbit as the two-dimensional plane spanned by two linearly independent vectors in $\mathbb{R}^{2} \times \mathbb{R}^{1} \times \mathbb{R}^{1}$ (see [20] for details). The first vector is given by $\left\{\left(D_{x} H, 0\right)\right\}$, which has to be evaluated at a given point $u^{ \pm} . D_{x}$ is the gradient in $(p, \phi)$ space and " 0 " denotes the two-dimensional zero vector. The second vector $\{\widetilde{J}\}$ is a unit vector parallel to the $J$ coordinate axis, which has to be viewed as emanating from the point $u^{ \pm}$. By varying $u^{ \pm}$we move the twodimensional hyperplane $\Pi_{u \pm}$ along the homoclinic orbit $\Gamma^{ \pm}$, where at each point $u^{ \pm} \in \Gamma^{ \pm}$the stable and unstable manifolds intersect $\Pi_{u \pm}$ transversely.

Since the level surfaces $h_{\epsilon}$ are preserved and the direction $\left(D_{x} H, 0\right)$ is complementary to these surfaces, the manifolds cannot split along this direction. Thus we do not need to measure along this direction. The signed distance $d_{\epsilon}$ at a point $u^{ \pm}$between $W^{s}\left(T_{\epsilon}\right)$ and $W^{u}\left(T_{\epsilon}\right)$ along the remaining direction on $\Pi_{u \pm}$ is then determined by the Melnikov function $M\left(\psi^{0}, t_{0}, \alpha\right)$ measuring the distances in $\left[J\left(W_{\epsilon}^{s}\right)-J\left(W_{\epsilon}^{u}\right)\right]$ up to order $\mathcal{O}\left(\epsilon^{2}\right)$.

Our trimer system belongs to category III of Wiggins classification in [20] with $n=m=1$. According to Wiggins [20] the Melnikov function is given by the following Melnikov integral:

$M^{ \pm}\left(\psi^{0}, t_{0}, \alpha\right)=-\int_{-\infty}^{\infty} d t D_{\psi} H^{1}\left[\Gamma^{ \pm}\left(t, \psi^{0}, t_{0}, \alpha\right)\right]$,

where $\Gamma^{ \pm}$denotes the homoclinic orbit for the unperturbed system given by Eqs. (35)-(41).

The Melnikov integral is then determined by

$$
M^{ \pm}\left(\psi^{0}, t_{0}, \alpha\right)=-\int_{-\infty}^{\infty} d t D_{\psi} H^{1}\left[p^{ \pm, h}\left(t-t_{0}\right), \phi^{h}\left(t-t_{0}\right) ; \bar{J}, \psi^{h}(t)+\psi^{0}\right] .
$$

We note that the $t_{0}$ dependence in the arguments of the Melnikov function can be eliminated, so that we are allowed to set $t_{0}=0$ in the following (see Chap. 4 in [20] for details). Further we restrict the analysis to the "+" sign in Eq. (43) since the case of the "- " sign can be treated on an equal footing.

Using Eq. (33) we obtain after some algebra

$$
M\left(\psi^{0}, \alpha\right)=-\epsilon \frac{W}{2} \sqrt{I^{2}-J^{2}} \int_{-\infty}^{\infty} d t\left\{F_{+}\left[p^{h}(t)\right] \sin \left[\psi^{h}(t)+\psi^{0}\right]+F_{-}\left[p^{h}(t)\right] \cos \left[\psi^{h}(t)+\psi^{0}\right]\right\},
$$

with

$$
F_{ \pm}\left[p^{h}(t)\right]=\sqrt{\left(1-\left[p^{h}(t)\right]^{2} \pm\left\{1-a\left[p^{h}(t)\right]^{2}\right\}\right)^{1 / 2}}\left[\left(\frac{1 \pm p^{h}(t)}{1 \mp p^{h}(t)}\right]^{1 / 4} \pm\left(\frac{1 \mp p^{h}(t)}{1 \pm p^{h}(t)}\right]^{1 / 4}\right],
$$

where $a=\alpha / A$ and $p^{h}(t)$ is given in Eq. (36) with the "+" sign. tion.

We are not able to compute the Melnikov integral explicitly, but we can show the validity of the following proposi-

Proposition. The Melnikov integral in Eq. (44) is of the form

$$
M\left(\psi^{0}, \alpha\right)=\widetilde{F}_{+}(W, V, I, \bar{J}, \gamma) \sin \left(\psi^{0}\right)+\widetilde{F}_{-}(W, V, I, \bar{J}, \gamma) \cos \left(\psi^{0}\right),
$$

where $\widetilde{F}_{ \pm}$are smooth functions of the system parameters.

Proof. The Melnikov integral in Eq. (44) is only conditionally convergent. Therefore we consider the improper integral as a limit of a sequence (see Chap. 4 in [20]). Expanding $\sin \left[\psi^{h}(t)+\psi^{0}\right]=\sin \left[\psi^{h}(t)\right] \cos \left(\psi^{0}\right)+\cos \left[\psi^{h}(t)\right] \sin \left(\psi^{0}\right)$ and $\cos \left[\psi^{h}(t)+\psi^{0}\right]=\cos \left[\psi^{h}(t)\right] \cos \left(\psi^{0}\right)-\sin \left[\psi^{h}(t)\right] \sin \left(\psi^{0}\right)$ and taking further into account that $\psi^{h}(t)$ is an odd function, the odd part of the integral can be omitted since it integrates to zero and we obtain for the even part of the integral

$$
\begin{aligned}
M\left(\psi^{0}, \alpha\right) & =-\epsilon \frac{W}{2} \sqrt{I^{2}-J^{2}} \int_{-\infty}^{\infty} d t\left\{F_{+}\left[p^{h}(t)\right] \sin \left(\psi^{0}\right)+F_{-}\left[p^{h}(t)\right] \cos \left(\psi^{0}\right)\right\} \cos \left[\psi^{h}(t)\right] \\
& \equiv-\epsilon \frac{W}{2} \sqrt{I^{2}-J^{2}}\left[M_{+} \sin \left(\psi^{0}\right)+M_{-} \cos \left(\psi^{0}\right)\right]
\end{aligned}
$$

Next we consider the integrals as a limit

$$
\begin{aligned}
M_{ \pm} & =\int_{-\infty}^{\infty} d t F_{ \pm}\left[p^{h}(t)\right] \cos \left[\psi^{h}(t)\right] \\
& =\lim _{n \rightarrow \infty} \int_{-2 \pi n / \omega}^{2 \pi n / \omega} d t\{\cos (\omega t) \cos [\Omega(t)]-\sin (\omega t) \sin [\Omega(t)]\} F_{ \pm}\left[p^{h}(t)\right]
\end{aligned}
$$

where we set

$$
\begin{aligned}
& \omega=-\frac{1}{8} \gamma(I+3 \bar{J})+\frac{1}{4 N} V(I-\bar{J}), \\
& \Omega(t)=\frac{1}{2 \gamma N^{2}} V(I-\bar{J})\left[\frac{\gamma N}{4 V}-1\right] \frac{1}{\sqrt{1-A^{2}}} \arctan \left[\left(\frac{A^{2}}{1-A^{2}}\right]^{1 / 2} \tanh (2 \alpha V t)\right] .
\end{aligned}
$$


and $\boldsymbol{A}$ is given in Eq. (39).

After integrating once by parts we are left with

$$
M_{ \pm}=\lim _{n \rightarrow \infty}\left\{-\frac{1}{\omega} \int_{-2 \pi n / \omega}^{2 \pi n / \omega} d t\left\{\cos [\omega t+\Omega(t)] \Omega^{\prime}(t) F_{ \pm}\left[p^{h}(t)\right]+\sin [\omega t+\Omega(t)] F_{ \pm}^{\prime}\left[p^{h}(t)\right]\right\}\right\},
$$

and the prime (') stands for the derivative $d / d t$.

We obtain

$$
\Omega^{\prime}(t)=\frac{V}{4 N}(I-\bar{J})\left[\frac{\gamma N}{4 V}-1\right] \frac{1}{\left[\frac{\gamma N}{4 \alpha V}\right]^{2} \cosh ^{2}(2 \alpha V t)-1},
$$

and

$$
\begin{aligned}
& F_{ \pm}^{\prime}\left[p^{h}(t)\right]=\mp \frac{1}{2}\left\{\frac{F_{ \pm}\left[p^{h}(t)\right]}{\sqrt{1-\left[p^{h}(t)\right]^{2} \pm\left\{1-a\left[p^{h}(t)\right]^{2}\right\}}}\left[2 a \pm \frac{1}{\sqrt{1-\left[p^{h}(t)\right]^{2}}}\right] p^{h}(t)\right. \\
& -\left(\sqrt{1-\left[p^{h}(t)\right]^{2}} \pm\left\{1-a\left[p^{h}(t)\right]^{2}\right\}\right)^{1 / 2} \frac{1}{\left\{1-\left[p^{h}(t)\right]^{2}\right\}} \\
& \left.\times\left[\left[\frac{1 \pm p^{h}(t)}{1 \mp p^{h}(t)}\right]^{1 / 4} \mp\left[\frac{1 \mp p^{h}(t)}{1 \pm p^{h}(t)}\right]^{1 / 4}\right]\right\}\left[\frac{8 \alpha^{2} V^{2}}{\gamma N}\right] \frac{\sinh (2 \alpha V t)}{\cosh ^{2}(2 \alpha V t)} \text {. }
\end{aligned}
$$

Upon inserting the above expressions for $\Omega^{\prime}$ and $F_{ \pm}^{\prime}$ into Eq. (51) we eventually get convergent integrals yielding $\widetilde{F}_{ \pm}(W, V, I, \bar{J}, \gamma)$.

The Melnikov function has simple zeros showing the presence of homoclinic chaos in the dynamics of the coupled system. Due to the resulting nonintegrable motions the stable and unstable manifolds of the periodic orbit of $M_{\epsilon}$ are no longer identical, but intersect each other, thus establishing a homoclinic tangle in the vicinity of the disturbed homoclinic orbit. Moreover, on a fixed energy manifold with chosen $\bar{J}$ an isolated one-torus $T_{\epsilon}(\bar{J})$ survives and is normally hyperbolic on the energy manifold. Hence our results for the Melnikov function show that the perturbed system contains transverse homoclinic orbits to a hyperbolic orbit and we have, due to the SmaleBirkhoff homoclinic theorem [20,27], homoclinic chaos in the coupled dynamics.

\section{SUMMARY}

In this paper an analytical proof is given of the existence of homoclinic chaos in the DST trimer by means of the generalized Melnikov method. Although not explicitly given in the present paper the nonintegrability of the DST trimer was proved by applying Ziglin's theorem [31].

Starting from the DST dimer as the underlying integrable subsystem the coupling to the third trimer oscillator has been treated perturbationally. The symmetry properties of the coupled dimer-oscillation system allows a reduction to a two degree of freedom Hamiltonian system. In analyzing the phase space structure special attention was payed to the homoclinic manifold. The existence of an infinite number of zeros of the Melnikov function was shown, which in turn guarantees the presence of a homoclinic tangle around the destructed homoclinic orbit.

\section{ACKNOWLEDGMENTS}

One of the authors (D.H.) gratefully acknowledges the support of the Technical University of Denmark. This work was supported by the Deutsche Forschungsgemeinschaft via Sonderforschungsbereich 337.
[1] J. C. Eilbeck, A. C. Scott, and P. S. Lomdahl, Physica D 16, 318 (1985).

[2] V. M. Kenkre and D. K. Campbell, Phys. Rev. B 34, 4595 (1986).

[3] A. S. Davydov and N. I. Kislukha, Phys. Status Solidi B 59, 465 (1973).

[4] N. Finlayson and G. I. Stegeman, Appl. Phys. Lett. 56, 2276 (1990).
[5] Y. Chen, A. W. Snyder, and D. J. Mitchell, Electron. Lett. 26, 77 (1990).

[6] J. H. Jensen, P. L. Christiansen, J. N. Elgin, J. D. Gibbon, and O. Skovgaard, Phys. Lett. 110A, 429 (1985).

[7] S. De Filippo, M. Fusco Girard, and M. Salerno, Physica D 26, 411 (1987).

[8] S. De Filippo, M. Fusco Girard, and M. Salerno, Physica D 29, 421 (1988). 
[9] S. De Filippo and M. Salerno, Phys. Lett A 142, 479 (1989).

[10] L. Cruzeiro-Hansson, H. Feddersen, R. Flesch, P. L. Christiansen, M. Salerno, and A. C. Scott, Phys. Rev. B 42, 522 (1990).

[11] K. W. DeLong, J. Yumoto, and N. Finlayson, Physica D 54, 36 (1991).

[12] L. J. Bernstein, Opt. Commun. 94, 406 (1992).

[13] N. Finlayson, K. J. Blow, L. J. Bernstein, and K. W. DeLong, Phys. Rev. A 48, 3863 (1993).

[14] D. Hennig and B. Esser, Phys. Rev. A 46, 4569 (1992).

[15] D. Hennig, J. Phys. A: Math. Nucl. Gen. 25, 1247 (1992).

[16] D. Hennig, Physica D 64, 121 (1993).

[17] P. L. Christiansen, M. F. Jørgensen, and V. B. Kuznetsov, Lett. Math. Phys. 29, 165 (1994).

[18] V. K. Melnikov, Trans. Moscow Math. Soc. 12, 1 (1963).

[19] J. Guckenheimer and P. Holmes, Nonlinear Oscillations, Dynamical Systems, and Bifurcations of Vector Fields (Springer-Verlag, New York, 1983).

[20] S. Wiggins, Global Bifurcations and Chaos-Analytical Methods (Springer-Verlag, New York, 1988); S. Wiggins,
Physica D 44, 471 (1990).

[21] G. Morrison (unpublished).

[22] G. Morrison (unpublished).

[23] R. Abraham and J. Marsden, Foundation of Mechanics (Addison-Wesley, Reading, MA 1978).

[24] K. R. Meyer and G. R. Hall, Introduction to Hamiltonian Dynamical Systems and the $N$-Body Problem (SpringerVerlag, New York, 1991).

[25] S. Wiggins, Chaotic Transport in Dynamical Systems (Springer-Verlag, New York, 1991).

[26] P. J. Holmes and J. E. Marsden, Commun. Math. Phys. 82, 523 (1982).

[27] S. Smale, in Differential and Combinatorial Topology, edited by S. S. Cairns (Princeton University Press, Princeton, NJ, 1963), pp. 63-80.

[28] N. Fenichel, Indiana Univ. Math. J. 21, 193 (1971).

[29] V. I. Arnold, Mathematical Methods of Classical Mechanics (Springer-Verlag, New York, 1978).

[30] S. M. Graff, J. Differ. Equations 15, 1 (1974).

[31] S. L. Ziglin, Trans. Moscow Math. Soc. 1, 283 (1982). 